\title{
Influence de la mise à jeun sur l'aminoacidémie libre, l'urémie et la glycémie chez la vache laitière
}

\author{
BB Ndibualonji ${ }^{*}$, D Dehareng ${ }^{2}$, JM Godeau $^{1}$ \\ ${ }^{1}$ Laboratoire de biochimie, faculté de médecine vétérinaire, université de Liège, \\ bd de Colonster, $n^{\circ} 20$, bât B42, B-4000 Liège-Sart Tilman ; \\ 2 Unité de génétique, faculté des sciences agronomiques, université catholique de Louvain, \\ place Croix-du-Sud, 2, bte 14, Louvain-la-Neuve, Belgique
}

(Reçu le 11 avril 1996 ; accepté le 24 octobre 1996)

\begin{abstract}
Summary - Effects of starvation on plasma amino acids, urea and glucose in dairy cows. Three cows within their 26th week of lactation (average milk yield: $15.7 \mathrm{~kg} /$ day) were used to determine the effects of starvation on 19 free amino acids (AA) of plasma, uremia and glycemia. For three successive days, blood samples were collected at hourly intervals from $09.00 \mathrm{am}$ to $03.00 \mathrm{pm}$. From 1 month before up to the 1 st day of the sampling period, the animals were overfed in two equal meals $(06.15$ am and $03.30 \mathrm{pm})$ with a hay-based diet. On the 2 nd and the $3 \mathrm{rd}$ day, only water was available. On the 1 st day of fasting, muscle protein catabolism increased as suggested by an increase $(P<0.05)$ in mean plasma levels of 3 -methylhistidine (MeHis, index of muscle protein catabolism in cattle), Asp, Gly, His, Lys and Ser. On the same day, mean plasma concentrations of Glu and Pro decreased $(P<0.05)$, possibly through an enhanced utilization of those AA for gluconeogenesis and/or energy needs as suggested by the maintainance of glycemia and the increase $(P<0.05)$ in uremia. On the 2 nd day of fasting, further changes of metabolic parameters occurred: mean plasma levels of MeHis, urea, Arg and Leu still increased $(P<0.05)$; those of Asn, Asp, Glu, Met and Tyr decreased $(P<0.05)$; plasma Gly, His, Lys and Ser plateaued at high and plasma Pro at low levels. Unlike results reported in dry cow, plasma Ala, Gln, Ile and Val did not increase during the fasting period. Furthermore, despite an enhanced protein mobilization, glycemia and milk yield dropped $(P<0.05)$ on the 2 nd day of fasting. The fall in plasma glucose probably resulted from a continuous glucose uptake by the mammary gland for milk lactose synthesis which was not entirely compensated by gluconeogenesis. Within-day variations in plasma metabolites were observed, but these variations were generally lower during fasting than during feeding, especially for plasma glucose which showed variation with time of day during both fasting days. It was concluded that, as in dry cows, a 2-day fasting enhances muscle proteolysis in lactating cows. Contrary to dry cows, however, gluconeogenesis mechanism which attempts to maintain glucose homeostasis becomes insufficient in lactating cows to avoid a drop in glycemia from the 2 nd day of fasting. The decrease in milk yield might be, at least partly, the result of that drop in glycemia. Finally, the time course from the last meal is a factor affecting within-day variations of plasma metabolites.
\end{abstract}

fasting / aminoacidemia / uremia / glycemia / dairy cattle

\footnotetext{
* Correspondance et tirés à part
} 
Résumé - Trois vaches en $26^{\mathrm{e}}$ semaine de lactation ont été utilisées pour déterminer les effets de la mise à jeun sur l'aminoacidémie libre, l'urémie et la glycémie. Les échantillons de sang ont été prélevés d'heure en heure pendant $3 \mathrm{j}$ successifs, de $9 \mathrm{~h}$ à $15 \mathrm{~h}$. Jusqu'au $1^{\text {er }}$ jour de mesures inclus, les animaux étaient alimentés $(6 \mathrm{~h} 15$ et $15 \mathrm{~h} 30)$ au-delà de leurs besoins. Les $2^{\mathrm{e}}$ et $3^{\mathrm{e}}$ jours, seule l'eau de boisson était disponible. Au $1^{\text {er }}$ jour de jeûne, une augmentation du catabolisme des protéines musculaires, appréciée par une augmentation $(p<0,05)$ de la teneur plasmatique moyenne en 3méthylhistidine (MeHis), a été observée. En outre, un accroissement $(p<0,05)$ des concentrations moyennes en urée, Asp, Gly, His, Lys et Ser, une diminution $(p<0,05)$ de celles en Glu et Pro ainsi que le maintien de la glycémie et de la production laitière ont été observés. Le $2^{\mathrm{e}}$ jour de jeûne s'est caractérisé par des modifications d'un plus grand nombre des paramètres étudiés, notamment par un accroissement $(p<0,05)$ des niveaux moyens en MeHis, urée, Arg et Leu, par une diminution $(p<0,05)$ de ceux en Asn, Asp, Glu, Met et Tyr, et par un maintien à des valeurs hautes de la Gly, His, Lys et Ser, et à des valeurs basses de la Pro. Malgré cette accentuation apparente de la mobilisation protéique, la glycémie et la production laitière ont chuté au cours du $2^{\mathrm{e}}$ jour de jeûne. Par ailleurs, des variations intrajournalières ont été observées mais, comparativement à la situation nourrie, elles ont été généralement faibles pendant le jeûne pour tous les paramètres métaboliques étudiés, et particulièrement pour la glycémie. Il a été conclu que la vache en lactation mobilise rapidement ses protéines musculaires au cours du jeûne. Cependant, le mécanisme de la néoglucogenèse ne permet d'assurer l'homéostasie glucidique qu'au cours des 24 premières heures de jeûne. La chute de la glycémie serait, du moins en partie, responsable de la chute de la production laitière au $2^{\mathrm{e}}$ jour de jeûne. Finalement, le temps écoulé depuis le dernier repas est un facteur affectant les variations intra-journalières de l'aminoacidémie, de l'urémie et de la glycémie.

jeûne / aminoacidémie / urémie / glycémie / vache laitière

\section{INTRODUCTION}

Lorsque la sous-alimentation devient sévère, la vache à l'entretien mobilise ses réserves protéiques en vue de satisfaire ses besoins en substrats néoglucogéniques et/ou énergétiques (Ndibualonji et al, 1992). Ainsi, chez les ruminants à l'entretien, les niveaux du glucose sanguin varient très peu au cours des premiers jours de jeûne (Bouchat et al, 1981 ; Ndibualonji et al, 1993). Cette normoglycémie est particulièrement importante pour la survie des animaux car, contrairement aux observations faites chez le monogastrique (rat : Hawkins et al, 1971 ; chien : Weiner et al, 1971), le cerveau des ruminants utilise principalement le glucose et très peu les corps cétoniques pour son métabolisme énergétique, que ce soit en situation de jeûne ou en situation nourrie (Lindsay et Setchell, 1976 ; Pell et Bergman, 1983).
Par ailleurs, la situation alimentaire de la vache est souvent critique en début de lactation, surtout lorsque la production laitière est importante. La synthèse du lait nécessite souvent plus d'énergie et d'acides aminés (AA) que ceux fournis par les quantités d'aliments que l'animal est capable d'ingérer. La réponse de l' animal se traduit notamment par une mobilisation de ses protéines musculaires (Baldwin et Kim, 1993), ce qui ne parvient pas toujours à maintenir la glycémie, la mamelle utilisant plus de $80 \%$ du glucose total de l'organisme chez la vache (Bickerstaffe et al, 1974). Ainsi une chute de la glycémie est fréquemment observée chez la vache en début de lactation (Baldwin et Kim, 1993).

Chez la vache en milieu de lactation, la glycémie semble peu varier, mais les informations à ce sujet sont très parcellaires (Ndibualonji, 1995). L'objectif du présent essai est donc de voir si une sous-alimentation 
sévère modifie ( $\mathrm{cf}$ vaches en début de lactation) ou non (cf vaches à l'entretien) le profil de la glycémie en milieu de lactation. De plus, l'impact du jeûne sur les niveaux moyens et l'évolution journalière des teneurs plasmatiques en AA libres et en urée a été évalué.

\section{MATÉRIEL ET MÉTHODES}

D’un poids moyen de $523( \pm 23) \mathrm{kg}$ et produisant en moyenne $15,7( \pm 1,2) \mathrm{kg} / \mathrm{j}$ de lait à $4 \%$ de matières grasses, trois vaches adultes de race Frisonne et en $26^{\mathrm{e}}( \pm 2)$ semaine de lactation ont été utilisées. Durant le mois précédant le début de l'essai, elles ont reçu une ration à base de foin de prairie naturelle et d'aliment concentré du commerce. Distribuée en deux repas quotidiens égaux (6h15-15h30), cette ration couvrait largement les besoins d'entretien et de production des animaux (tableau I). L'eau de boisson était disponible en permanence pendant la durée de l'essai. Réalisée à l'aide d'une machine mobile, la traite était effectuée deux fois par jour pendant les repas. Pour chaque animal, le lait des deux traites journalières était rassemblé, mélangé et pesé.

La veille du jour des prélèvements des échantillons de sang, un cathéter a été placé sous anesthésie locale dans la veine jugulaire de chaque vache. Le lendemain, de $9 \mathrm{~h}$ à $15 \mathrm{~h}$, les échantillons de sang $(5 \mathrm{~mL})$ ont été prélevés d'heure en heure à l'aide d'une seringue appliquée à l'extrémité du cathéter intrajugulaire. Ces prélèvements ont eu lieu pendant $3 \mathrm{j}$ successifs. Le premier jour, les animaux étaient nourris en deux repas égaux (situation témoin). Les repas étaient totalement consommés en 2 h environ (observations visuelles). Puis, les $2 \mathrm{j}$ suivants, seule l'eau de boisson était disponible. Le plasma destiné au dosage des AA libres a été immédiatement déprotéinisé à l'aide d'une solution d'acide trichloracétique à $5 \%$ (vol $/ \mathrm{vol})$. Tous les échantillons de plasma ont ensuite été congelés $\left(-20^{\circ} \mathrm{C}\right)$ jusqu'au moment du dosage des AA libres, de l'urée et du glucose.

La teneur des aliments en matière sèche, matière organique et azote total a été déterminée respectivement par dessication à l'étuve $\left(105^{\circ} \mathrm{C}\right)$, par incinération et par la méthode de Kjeldahl (Ndibualonji et al, 1995). La valeur azotée (protéines digestibles dans l'intestin grêle ou PDI) de la ration a été évaluée à partir des tables alimentaires (Andrieu et al, 1988). L'estimation de l'apport du régime en énergie nette du lait a été réalisée par collecte séparée des matières fécales et de l'urine pendant les $5 \mathrm{j}$ qui précédaient le début des prélèvements sanguins (Dehareng et Godeau, 1991). L'urémie a été déterminée selon une méthode à la thiosemicarbazide adaptée au dosage de routine sur un Auto-Analyzer Technicon (Ndibualonji, 1995). Les concentrations des 19 AA libres du plasma (MeHis, Ala, Arg,

Tableau I. Niveau d'ingestion et composition moyenne de la ration précédant le jeûne $(n=3)$.

Quantités ingérées ( $\mathrm{kg} M S / \mathrm{j})$

Foin de prairie naturelle

Pulpes sèches de betterave

Concentré ${ }^{1}$

\section{Composition chimique}

Matière organique $(\mathrm{g} / \mathrm{kg} \mathrm{MS})$

921

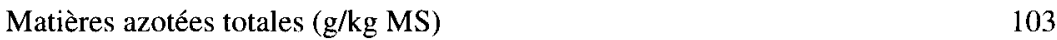

Énergie brute (MJ/kg MS)

Apports

UFL ( $\%$ des besoins)

132

PDI (\% des besoins)

146

\footnotetext{
${ }^{1}$ Aliment concentré du commerce (Moulins Dedobbeleer, Halle-Belgique) à $18 \%$ de protéines brutes.
} 
Asn, Asp, Glu, Gln, Gly, His, Ile, Leu, Lys, Met, Phe, Pro, Ser, Thr, Tyr et Val) ont été déterminées par HPLC (Ndibualonji et al, 1992). La mesure du glucose plasmatique a été effectuée par une méthode colorimétrique à l'hexokinase (Schmidt, 1961). Tous ces paramètres métaboliques ont été mesurés dans chaque échantillon prélevé, soit sept échantillons par vache et par jour.

Les résultats sont présentés sous forme de moyenne \pm écart type (écarts types obtenus à partir des sept moyennes horaires pour l'étude des niveaux moyens journaliers et à partir de chaque moyenne horaire pour l'étude de l'évolution journalière). Les différences statistiques entre les jours de mesure et entre les heures de prélèvement ont été étudiées en utilisant une analyse de la variance pour mesures répétées (Sas, 1989).

\section{RÉSULTATS}

\section{Niveaux moyens journaliers}

Le $1^{\text {er }}$ jour de jeûne, soit de la $18^{\mathrm{e}}$ à la $24^{\mathrm{e}}$ heure après le dernier repas, s'est traduit par une augmentation significative $(p<0,05)$ des teneurs plasmatiques en urée, MeHis, Asp, Gly, His, Lys et Ser, par une diminution $(p<0,05)$ de celles en Glu et Pro, et par le maintien de la glycémie, du poids vif et de la production laitière (tableau II).

Au cours du deuxième jour de jeûne, soit de la $42^{\mathrm{e}}$ à la $48^{\mathrm{e}}$ heure après le dernier repas, de nouvelles modifications métaboliques sont venues s'ajouter à celles du $1^{\mathrm{er}}$ jour. En effet, si l'urémie et la MeHis du plasma ont continué à augmenter $(p<0,05)$ et les concentrations plasmatiques du Glu à diminuer $(p<0,05)$, celles de l'Asp sont retournées $(p<0,05)$ à leur niveau initial (situation nourrie) tandis que celles de la Gly, de l'His, de la Lys, de la Pro et de la Ser se sont maintenues au niveau enregistré la veille; en revanche, les teneurs plasmatiques en glucose, Met et Thr ont chuté $(p<0,05)$ alors que celles en Arg et Leu ont augmenté $(p<0,05)$. Par ailleurs, le poids vif moyen et la production laitière des animaux ont chuté $(p<0,05)$ pendant le dernier jour de jeûne (tableau II).

\section{Évolution journalière des concentrations plasmatiques}

À l'état nourri, les concentrations plasmatiques en certains métabolites ont graduellement augmenté environ $1 \mathrm{~h}$ après la fin du repas $(9 \mathrm{~h})$ pour atteindre des niveaux plus élevés $(P<0,05)$ aux heures les plus éloignées de la fin du repas, c'est-à-dire à partir de la $2^{\mathrm{e}}$ heure pour la Gly, la Leu et la Val (sauf à $11 \mathrm{~h}$ ), de la $3^{\mathrm{e}}$ heure pour l'Arg, l'Ile et l'His (sauf à $13 \mathrm{~h}$ ), de la $4^{\mathrm{e}}$ heure pour l'Ala, la Met (sauf à 14. h) et la Phe, ainsi qu'à partir de la $6^{\mathrm{e}}$ heure qui suit la fin de l'ingestion des aliments pour la MeHis (tableaux III, IV, V). À l'inverse, la teneur plasmatique en d'autres métabolites s'est maintenue ou s'est légèrement accrue avant de chuter $(p<0,05)$ aux heures les plus éloignées du repas, soit à partir de la $4^{\mathrm{e}}$ heure pour l'Asp et la Tyr, de la $6^{\mathrm{e}}$ heure pour l'Asn et de la $7^{\mathrm{e}}$ heure après la fin du repas pour la Gln et la Thr (tableaux III, IV, V). Enfin, à l'exception du Glu dont la concentration a augmenté $(p<0,05)$ de la $2^{\mathrm{e}}$ à la $5^{\mathrm{e}}$ heure après la fin du repas avant de rejoindre son niveau initial, les autres métabolites, en particulier le glucose (sauf à $14 \mathrm{~h}$ ), n'ont pratiquement pas varié au fil du temps écoulé depuis la fin du repas (tableaux III, IV, V).

En situation de jeûne, si des modifications dans les concentrations de tous les paramètres métaboliques étudiés ont été observées, leur ampleur a généralement été plus faible qu'en situation nourrie. Ainsi aucune évolution intrajournalière n'a été, par exemple, observée le $1^{\text {er }}$ jour de jeûne dans les concentrations plasmatiques en urée, Ala et Lys, et le $2^{\mathrm{e}}$ jour de jeûne dans les concentrations en His, Ile, Ser et Val (tableaux III, IV, V). En particulier, aucune évolution intrajournalière significative n'a 
Tableau II. Évolution moyenne du poids vif, de la production laitière, de l'urémie, de la glycémie et de l'aminoacidémie libre au cours du jeûne chez trois vaches laitières.

\begin{tabular}{|c|c|c|c|}
\hline & \multirow[t]{2}{*}{ Nourries } & \multicolumn{2}{|c|}{ Jê̂ne } \\
\hline & & Jour 1 & Jour 2 \\
\hline Poids vif (kg) & $523 \pm 23^{\mathrm{a}}$ & $502 \pm 28^{\mathrm{ab}}$ & $459 \pm 20^{\mathrm{b}}$ \\
\hline Production laitière $^{\mathrm{l}}(\mathrm{kg} / \mathrm{j})$ & $16 \pm 1^{\mathrm{a}}$ & $15 \pm 1^{\mathrm{a}}$ & $7,8 \pm 1^{\mathrm{b}}$ \\
\hline Urémie (mg/l) & $298 \pm 19^{a}$ & $350 \pm 21^{b}$ & $436 \pm 28^{c}$ \\
\hline Glycémie (mg/100 ml) & $70 \pm 3^{a}$ & $73 \pm 3^{\mathrm{a}}$ & $61 \pm 3^{b}$ \\
\hline \multicolumn{4}{|c|}{ Aminoacidémie libre $(\mu \mathrm{mol} / \mathrm{l})$ : } \\
\hline 3-méthylhistidine & $10 \pm 1^{\mathrm{a}}$ & $14 \pm 1^{\mathrm{b}}$ & $19 \pm 1^{c}$ \\
\hline Alanine & $230 \pm 13$ & $267 \pm 16$ & $263 \pm 13$ \\
\hline Arginine & $93 \pm 5^{\mathrm{a}}$ & $102 \pm 6^{\mathrm{a}}$ & $149 \pm 8^{b}$ \\
\hline Asparagine & $47 \pm 2^{\mathrm{a}}$ & $44 \pm 2^{a b}$ & $35 \pm 3^{b}$ \\
\hline Aspartate & $22 \pm 1^{\mathrm{a}}$ & $30 \pm 1^{b}$ & $22 \pm 1^{a}$ \\
\hline Glutamate & $133 \pm 4^{\mathrm{a}}$ & $106 \pm 5^{b}$ & $85 \pm 4^{c}$ \\
\hline Glutamine & $284 \pm 17$ & $304 \pm 17$ & $285 \pm 18$ \\
\hline Glycine & $312 \pm 17^{\mathrm{a}}$ & $523 \pm 29^{b}$ & $582 \pm 32^{b}$ \\
\hline Histidine & $194 \pm 8^{\mathfrak{a}}$ & $247 \pm 12^{b}$ & $261 \pm 13^{\mathrm{b}}$ \\
\hline Isoleucine & $104 \pm 7$ & $124 \pm 7$ & $126 \pm 11$ \\
\hline Leucine & $118 \pm 9^{a}$ & $122 \pm 10^{\mathrm{a}}$ & $174 \pm 12^{b}$ \\
\hline Lysine & $81 \pm 3^{\mathrm{a}}$ & $115 \pm 5^{\mathrm{b}}$ & $115 \pm 4^{b}$ \\
\hline Méthionine & $22 \pm 1^{\mathrm{a}}$ & $19 \pm 1^{a}$ & $15 \pm 1^{b}$ \\
\hline Phénylalanine & $55 \pm 3$ & $46 \pm 3$ & $48 \pm 4$ \\
\hline Proline & $73 \pm 4^{a}$ & $57 \pm 1^{\mathrm{b}}$ & $53 \pm 4^{b}$ \\
\hline Sérine & $81 \pm 5^{\mathrm{a}}$ & $113 \pm 4^{b}$ & $129 \pm 7^{b}$ \\
\hline Thréonine & $78 \pm 2^{\mathrm{ab}}$ & $87 \pm 4^{\mathrm{a}}$ & $73 \pm 3^{b}$ \\
\hline Tyrosine & $40 \pm 3^{a}$ & $35 \pm 3^{\mathrm{ab}}$ & $27 \pm 2^{b}$ \\
\hline Valine & $216 \pm 18$ & $227 \pm 21$ & $248 \pm 15$ \\
\hline
\end{tabular}

1 Lait corrigé à $4 \%$ de matières grasses, ${ }^{a, b, c}$ Les moyennes dans une même ligne sans lettre commune sont différentes $(p<0,05)$.

été observée dans les niveaux de la glycémie pendant les deux jours de jeûne (tableau III).

\section{DISCUSSION}

\section{Niveaux moyens journaliers}

Globalement, les résultats enregistrés dans le sang au cours des 24 premières heures de jeûne sont en accord avec nos observations préliminaires chez la vache tarie (Ndibualonji et al, 1992 et 1993). En particulier, cette mise à jeun s'est traduite par une mobilisation des protéines musculaires, comme le suggère l'augmentation des teneurs plasmatiques en MeHis et en d'autres AA (Asp, Gly, His, Lys, Ser). En effet, chez la chèvre tarie (Nagasawa et al, 1993) comme chez la vache tarie ou en lactation (Ndibualonji, 1995), la MeHis du plasma est, au même titre que la quantité de MeHis excrétée dans 
Tableau III. Évolution journalière de la 3-méthylhistidine, de l'urée et du glucose du plasma chez trois vaches en lactation nourries, puis soumises à un jeûne complet de $2 \mathrm{j}$.

\begin{tabular}{lccccccc}
\hline & $9 h$ & $10 h$ & $11 h$ & $12 h$ & $13 h$ & $14 h$ & $15 h$ \\
\hline 3-méthylhistidine & & & & & & & \\
Nourries & $8 \pm 1^{\mathrm{a}}$ & $9 \pm 1^{\mathrm{ab}}$ & $9 \pm 1^{\mathrm{ab}}$ & $10 \pm 1^{\mathrm{b}}$ & $9 \pm 1^{\mathrm{ab}}$ & $12 \pm 1^{\mathrm{c}}$ & $13 \pm 1^{\mathrm{c}}$ \\
Jeûne j 1 & $12 \pm 1^{\mathrm{a}}$ & $12 \pm 1^{\mathrm{a}}$ & $14 \pm 2^{\mathrm{bc}}$ & $13 \pm 2^{\mathrm{ab}}$ & $17 \pm 3^{\mathrm{c}}$ & $15 \pm 2^{\mathrm{bc}}$ & $17 \pm 3^{\mathrm{c}}$ \\
Jeûne j 2 & $19 \pm 3^{\mathrm{ac}}$ & $15 \pm 1^{\mathrm{b}}$ & $22 \pm 4^{\mathrm{ad}}$ & $14 \pm 3^{\mathrm{b}}$ & $16 \pm 3^{\mathrm{bc}}$ & $24 \pm 4^{\mathrm{d}}$ & $22 \pm 3^{\mathrm{ad}}$ \\
& & & & & & & \\
Urée & & & & & & & \\
Nourries & $341 \pm 36^{\mathrm{a}}$ & $330 \pm 32^{\mathrm{ab}}$ & $291 \pm 18^{\mathrm{bc}}$ & $267 \pm 24^{\mathrm{c}}$ & $269 \pm 26^{\mathrm{c}}$ & $309 \pm 26^{\mathrm{ac}}$ & $281 \pm 30^{\mathrm{bc}}$ \\
Jeûne j 1 & $355 \pm 29$ & $338 \pm 34$ & $331 \pm 32$ & $326 \pm 22$ & $355 \pm 29$ & $370 \pm 33$ & $379 \pm 44$ \\
Jeûne j 2 & $418 \pm 39^{\mathrm{ab}}$ & $431 \pm 32^{\mathrm{ab}}$ & $415 \pm 37^{\mathrm{a}}$ & $453 \pm 43^{\mathrm{ab}}$ & $464 \pm 41^{\mathrm{b}}$ & $435 \pm 41^{\mathrm{ab}}$ & $444 \pm 40^{\mathrm{ab}}$ \\
& & & & & & & \\
Glucose & & & & & & & \\
Nourries & $65 \pm 4^{\mathrm{a}}$ & $69 \pm 5^{\mathrm{ab}}$ & $67 \pm 6^{\mathrm{ab}}$ & $74 \pm 5^{\mathrm{ab}}$ & $71 \pm 6^{\mathrm{ab}}$ & $76 \pm 6^{\mathrm{b}}$ & $67 \pm 5^{\mathrm{ab}}$ \\
Jeûne j 1 & $74 \pm 4$ & $77 \pm 5$ & $69 \pm 6$ & $73 \pm 4$ & $72 \pm 5$ & $70 \pm 3$ & $76 \pm 6$ \\
Jeûne j 2 & $58 \pm 5$ & $62 \pm 4$ & $65 \pm 4$ & $58 \pm 4$ & $63 \pm 3$ & $64 \pm 5$ & $58 \pm 4$ \\
\hline
\end{tabular}

Les repas ont été distribués à $6 \mathrm{~h} 15$ et à $15 \mathrm{~h} 30$. a,b,c,d,c Les moyennes dans une même ligne sans lettre commune sont différentes $(p<0,05)$.

les urines, un index du catabolisme des protéines musculaires pendant le jeûne. Par ailleurs, les réductions des teneurs en Glu et Pro du plasma suggèrent une augmentation de la synthèse du glucose à partir de ces composés qui sont tous glucoformateurs chez les ruminants (Ndibualonji et Godeau, 1993). Cela permet d'expliquer à la fois le maintien des valeurs physiologiques de la glycémie et l'augmentation de l'urémie pendant le $1^{\text {er }}$ jour de jeûne. Cette augmentation de la néoglucogenèse AA-dépendante dès le $1^{\text {er }}$ jour de jeûne est mise en évidence par l'étude de Lomax et Baird (1983) qui observent chez la vache que l'extraction hépatique du propionate passe de 4,98 $\mathrm{mmol} / \mathrm{min}$ en situation nourrie à 0,91 $\mathrm{mmol} / \mathrm{min}$ le $1^{\text {er }}$ jour de jeûne tandis que celle des AA passe de 0,36 à 1,72 $\mathrm{mmol} / \mathrm{min}$. Cependant, si l'utilisation rapide du Glu pendant le jeûne rejoint les observations faites chez le mouton (Leibholz,
1970 ; Heitmann et Bergman, 1980 ; Koenig et Boling, 1980), celle de la Pro semble être une particularité de la vache car elle n'a pas été observée dans les études précitées, même après un jeûne de $5 \mathrm{j}$. En revanche, la chute rapide de la prolinémie a déjà été rapportée en début de jeûne chez la vache (Lomax et Baird, 1983 ; Ndibualonji et al, 1992 ; Ndibualonji, 1995).

$\mathrm{Au} 2 \mathrm{e}$ jour de jeûne, une accentuation apparente de la mobilisation protéique (augmentation accrue de la MeHis du plasma) et des modifications d'un plus grand nombre de paramètres, notamment un accroissement des niveaux plasmatiques en urée, Arg et Leu, une diminution de celles en glucose, Asn, Asp, Glu, Met et Tyr, et un maintien à des valeurs hautes de la Gly, de l'His, de la Lys et de la Ser et à des valeurs basses de la Pro, ont été observées. La chute $(p<0,05)$ du poids vif observée seulement le $2^{\mathrm{e}}$ jour de jeûne confirme l'importance de la mobili- 
Tableau IV. Évolution journalière des acides aminés non essentiels chez trois vaches en lactation nourries, puis soumises à un jeûne complet de $2 \mathrm{j}$.

\begin{tabular}{cccccccc}
\hline & $9 h$ & $10 h$ & $11 h$ & $12 h$ & $13 h$ & $14 h$ & $15 h$ \\
\hline $\begin{array}{c}\text { Alanine } \\
\text { Nourries }\end{array}$ & $183 \pm 18^{\mathrm{a}}$ & $209 \pm 23^{\mathrm{a}}$ & $203 \pm 11^{\mathrm{a}}$ & $245 \pm 24^{\mathrm{b}}$ & $247 \pm 42^{\mathrm{b}}$ & $260 \pm 19^{\mathrm{b}}$ & $262 \pm 37^{\mathrm{b}}$ \\
Jeûne j 1 & $269 \pm 18$ & $263 \pm 22$ & $274 \pm 27$ & $259 \pm 26$ & $256 \pm 24$ & $276 \pm 18$ & $271 \pm 29$ \\
Jeûne j 2 & $256 \pm 16^{\mathrm{ab}}$ & $263 \pm 26^{\mathrm{ac}}$ & $244 \pm 23^{\mathrm{bc}}$ & $220 \pm 23^{\mathrm{b}}$ & $285 \pm 35^{\mathrm{a}}$ & $289 \pm 23^{\mathrm{a}}$ & $285 \pm 23^{\mathrm{a}}$ \\
& & & & & & & \\
Asparagine & & & & & & & \\
Nourries & $46 \pm 5^{\mathrm{ab}}$ & $54 \pm 8^{\mathrm{a}}$ & $53 \pm 4^{\mathrm{a}}$ & $55 \pm 6^{\mathrm{a}}$ & $51 \pm 8^{\mathrm{a}}$ & $37 \pm 6^{\mathrm{bc}}$ & $32 \pm 3^{\mathrm{c}}$ \\
Jeûne j 1 & $41 \pm 6^{\mathrm{a}}$ & $48 \pm 5^{\mathrm{a}}$ & $31 \pm 1^{\mathrm{b}}$ & $42 \pm 6^{\mathrm{a}}$ & $45 \pm 5^{\mathrm{a}}$ & $56 \pm 7^{\mathrm{c}}$ & $42 \pm 6^{\mathrm{a}}$ \\
Jeûne j 2 & $36 \pm 3^{\mathrm{ab}}$ & $36 \pm 6^{\mathrm{ab}}$ & $34 \pm 6^{\mathrm{ac}}$ & $40 \pm 5^{\mathrm{b}}$ & $35 \pm 5^{\mathrm{ab}}$ & $29 \pm 1^{\mathrm{c}}$ & $38 \pm 5^{\mathrm{ab}}$ \\
Aspartate & & & & & & & \\
Nourries & $27 \pm 4^{\mathrm{ab}}$ & $21 \pm 2^{\mathrm{ac}}$ & $29 \pm 5^{\mathrm{b}}$ & $22 \pm 4^{\mathrm{ac}}$ & $19 \pm 3^{\mathrm{c}}$ & $21 \pm 3^{\mathrm{ac}}$ & $16 \pm 3^{\mathrm{c}}$ \\
Jeûne J 1 & $24 \pm 4^{\mathrm{a}}$ & $34 \pm 5^{\mathrm{bc}}$ & $36 \pm 6^{\mathrm{b}}$ & $25 \pm 4^{\mathrm{a}}$ & $30 \pm 4^{\mathrm{ab}}$ & $35 \pm 6^{\mathrm{b}}$ & $26 \pm 3^{\mathrm{ac}}$ \\
Jeûne J 2 & $22 \pm 3^{\mathrm{ab}}$ & $26 \pm 4^{\mathrm{a}}$ & $21 \pm 3^{\mathrm{b}}$ & $23 \pm 3^{\mathrm{ab}}$ & $19 \pm 3^{\mathrm{b}}$ & $23 \pm 2^{\mathrm{ab}}$ & $21 \pm 3^{\mathrm{ab}}$
\end{tabular}

Glutamate

\begin{tabular}{|c|c|c|c|c|c|c|c|}
\hline Nourries & $115 \pm 11^{\mathrm{ac}}$ & $146 \pm 16^{b}$ & $135 \pm 15^{b}$ & $144 \pm 15^{b}$ & $142 \pm 13^{b}$ & $131 \pm 17^{a b}$ & $114 \pm 13^{c}$ \\
\hline & $104 \pm 14^{\mathrm{ab}}$ & $94 \pm 16^{a}$ & $95 \pm 19^{\mathrm{ad}}$ & $115 \pm 14^{b d}$ & $91 \pm 14^{a}$ & $129 \pm 10^{b c}$ & $118 \pm 12^{b}$ \\
\hline Jên & $85 \pm 10^{\mathrm{ab}}$ & $91 \pm 12^{\mathrm{a}}$ & $86 \pm 10^{\mathrm{ab}}$ & $80 \pm 6^{a b}$ & $90 \pm 9^{a}$ & $88 \pm 7^{\mathrm{a}}$ & $74 \pm 6^{b}$ \\
\hline
\end{tabular}

Glutamine

$\begin{array}{llllllll}\text { Nourries } & 277 \pm 21^{\mathrm{a}} & 307 \pm 24^{\mathrm{b}} & 292 \pm 19^{\mathrm{ab}} & 304 \pm 21^{\mathrm{ab}} & 297 \pm 24^{\mathrm{ab}} & 278 \pm 20^{\mathrm{a}} & 238 \pm 17^{\mathrm{c}} \\ \text { Jeûne j 1 } & 306 \pm 16^{\mathrm{ac}} & 286 \pm 19^{\mathrm{ab}} & 313 \pm 26^{\mathrm{ac}} & 271 \pm 23^{\mathrm{b}} & 300 \pm 23^{\mathrm{bc}} & 331 \pm 26^{\mathrm{c}} & 302 \pm 23^{\mathrm{c}} \\ \text { Jeûne j 2 } & 290 \pm 20^{\mathrm{a}} & 272 \pm 22^{\mathrm{ac}} & 286 \pm 19^{\mathrm{a}} & 335 \pm 31^{\mathrm{b}} & 282 \pm 24^{\mathrm{a}} & 281 \pm 24^{\mathrm{a}} & 248 \pm 19^{\mathrm{c}}\end{array}$

Glycine

$\begin{array}{llllllll}\text { Nourries } & 222 \pm 30^{\mathrm{a}} & 274 \pm 19^{\mathrm{b}} & 289 \pm 34^{\mathrm{bc}} & 320 \pm 29^{\mathrm{c}} & 363 \pm 37^{\mathrm{d}} & 352 \pm 24^{\mathrm{d}} & 364 \pm 23^{\mathrm{d}} \\ \text { Jeûne j 1 } & 422 \pm 31^{\mathrm{a}} & 482 \pm 31^{\mathrm{be}} & 451 \pm 45^{\mathrm{ae}} & 568 \pm 39^{\mathrm{c}} & 520 \pm 34^{\mathrm{b}} & 636 \pm 37^{\mathrm{d}} & 586 \pm 24^{\mathrm{c}} \\ \text { Jeûne j 2 } & 578 \pm 42^{\text {ac }} & 504 \pm 32^{\mathrm{b}} & 535 \pm 23^{\mathrm{ab}} & 596 \pm 34^{\mathrm{c}} & 653 \pm 26^{\mathrm{d}} & 612 \pm 32^{\text {cd }} & 590 \pm 29^{\mathrm{c}}\end{array}$

Proline

$\begin{array}{llllllll}\text { Nourries } & 64 \pm 11^{\mathrm{a}} & 73 \pm 8^{\mathrm{ab}} & 81 \pm 8^{\mathrm{b}} & 72 \pm 8^{\mathrm{ab}} & 72 \pm 10^{\mathrm{ab}} & 85 \pm 11^{\mathrm{b}} & 66 \pm 4^{\mathrm{a}} \\ \text { Jeûne j 1 } & 56 \pm 8^{\mathrm{ab}} & 57 \pm 7^{\mathrm{ab}} & 53 \pm 9^{\mathrm{ab}} & 63 \pm 8^{\mathrm{a}} & 49 \pm 10^{\mathrm{b}} & 60 \pm 8^{\mathrm{ab}} & 61 \pm 10^{\mathrm{ab}} \\ \text { Jeûne j 2 } & 59 \pm 5^{\mathrm{a}} & 68 \pm 10^{\mathrm{a}} & 57 \pm 5^{\mathrm{a}} & 56 \pm 8^{\mathrm{a}} & 34 \pm 6^{\mathrm{b}} & 57 \pm 6^{\mathrm{a}} & 43 \pm 6^{\mathrm{b}}\end{array}$

Sérine

$\begin{array}{lccccccc}\text { Nourries } & 80 \pm 7^{\mathrm{ab}} & 70 \pm 6^{\mathrm{a}} & 78 \pm 8^{\mathrm{ab}} & 84 \pm 6^{\mathrm{b}} & 83 \pm 9^{\mathrm{b}} & 85 \pm 7^{\mathrm{b}} & 89 \pm 10^{\mathrm{b}} \\ \text { Jeûne j 1 } & 86 \pm 9^{\mathrm{a}} & 90 \pm 10^{\mathrm{a}} & 118 \pm 15^{\mathrm{bd}} & 108 \pm 10^{\mathrm{b}} & 113 \pm 16^{\mathrm{b}} & 132 \pm 16^{\mathrm{a}} & 146 \pm 18^{\mathrm{c}} \\ \text { Jeûne j 2 } & 125 \pm 17 & 127 \pm 15 & 121 \pm 13 & 125 \pm 11 & 129 \pm 16 & 140 \pm 14 & 133 \pm 15\end{array}$

Les repas ont été distribués à $6 \mathrm{~h} 15$ et à 15 h 30 , a.b.c.,d,e Les moyennes dans une même ligne sans lettre commune sont différentes $(p<0,05)$. 
Tableau V. Évolution journalière des acides aminés essentiels chez trois vaches en lactation nourries, puis soumises à un jeûne complet de $2 \mathrm{j}$.

\begin{tabular}{|c|c|c|c|c|c|c|c|}
\hline Heures & $9 h$ & $10 h$ & $11 \mathrm{~h}$ & $12 h$ & $13 h$ & $14 h$ & $15 h$ \\
\hline \multicolumn{8}{|l|}{ Arginine } \\
\hline Nourries & $70 \pm 6^{a}$ & $76 \pm 6^{a}$ & $95 \pm 3^{b d}$ & $90 \pm 7^{\text {bd }}$ & $103 \pm 9^{c d}$ & $104 \pm 11^{\mathrm{cd}}$ & $110 \pm 12^{\mathrm{c}}$ \\
\hline Jeûne j I & $82 \pm 6^{\mathrm{ab}}$ & $77 \pm 7^{\mathrm{a}}$ & $91 \pm 8^{b}$ & $113 \pm 9^{c}$ & $106 \pm 10^{c}$ & $111 \pm 10^{c}$ & $135 \pm 12^{\mathrm{d}}$ \\
\hline Jeûne j 2 & $145 \pm 14^{\mathrm{ab}}$ & $135 \pm 16^{a}$ & $148 \pm 18^{a b}$ & $158 \pm 19^{b}$ & $150 \pm 15^{a b}$ & $150 \pm 16^{\mathrm{ab}}$ & $155 \pm 17^{\mathrm{ab}}$ \\
\hline \multicolumn{8}{|l|}{ Histidine } \\
\hline Nourries & $156 \pm 21^{a}$ & $168 \pm 13^{\text {ad }}$ & $208 \pm 22^{b c}$ & $198 \pm 17^{\text {bd }}$ & $181 \pm 14^{\mathrm{ab}}$ & $206 \pm 22^{b}$ & $244 \pm 24^{c}$ \\
\hline Jeûne j 1 & $245 \pm 25^{\mathrm{ab}}$ & $222 \pm 20^{b}$ & $237 \pm 23^{a b}$ & $227 \pm 13^{b}$ & $250 \pm 18^{\mathrm{ad}}$ & $291 \pm 28^{c}$ & $259 \pm 26^{\mathrm{ad}}$ \\
\hline Jeûne $\mathrm{j} 2$ & $251 \pm 22$ & $260 \pm 22$ & $254 \pm 25$ & $262 \pm 25$ & $261 \pm 30$ & $267 \pm 20$ & $273 \pm 28$ \\
\hline \multicolumn{8}{|l|}{ Isoleucine } \\
\hline Nourries & $72 \pm 14^{\mathrm{a}}$ & $80 \pm 13^{\mathrm{ab}}$ & $101 \pm 16^{\mathrm{bd}}$ & $122 \pm 18^{\mathrm{cd}}$ & $120 \pm 14^{\mathrm{cd}}$ & $109 \pm 15^{\mathrm{cd}}$ & $126 \pm 13^{c}$ \\
\hline Jeûne j 1 & $108 \pm 17^{\mathrm{a}}$ & $103 \pm 16^{\mathrm{a}}$ & $137 \pm 19^{\mathrm{b}}$ & $115 \pm 14^{a}$ & $149 \pm 14^{b}$ & $105 \pm 12^{\mathrm{a}}$ & $145 \pm 11^{b}$ \\
\hline Jeûne $\mathbf{j} 2$ & $115 \pm 14$ & 125 & $124 \pm 11$ & $131 \pm 17$ & $129 \pm 18$ & $132 \pm 17$ & $125 \pm 13$ \\
\hline \multicolumn{8}{|l|}{ Leucine } \\
\hline Nourries & $91 \pm 11^{\mathrm{a}}$ & $112 \pm 13^{b}$ & $107 \pm 16^{\mathrm{b}}$ & $127 \pm 20^{\mathrm{bc}}$ & $127 \pm 15^{\mathrm{c}}$ & $141 \pm 14^{c}$ & $125 \pm 21^{b c}$ \\
\hline Jeûne j 1 & $101 \pm 12^{\mathrm{a}}$ & $121 \pm 20^{b}$ & $97 \pm 12^{\mathrm{a}}$ & $147 \pm 25^{c}$ & $129 \pm 19^{b c}$ & $125 \pm 17^{b}$ & $131 \pm 13^{b c}$ \\
\hline Jeûne j 2 & $164 \pm 19^{\mathrm{ac}}$ & $155 \pm 19^{\mathrm{a}}$ & $167 \pm 21^{\mathrm{ac}}$ & $190 \pm 20^{\mathrm{b}}$ & $177 \pm 22^{b c}$ & $179 \pm 23^{b c}$ & $187 \pm 17^{\mathrm{b}}$ \\
\hline \multicolumn{8}{|l|}{ Lysine } \\
\hline Nourries & $90 \pm 10^{\mathrm{ad}}$ & $82 \pm 6^{\text {ae }}$ & $72 \pm 8^{b c}$ & $65 \pm 8^{b}$ & $97 \pm 12^{d}$ & $80 \pm 9^{c e}$ & $83 \pm 6^{\mathrm{ae}}$ \\
\hline Jeûne j 1 & $110 \pm 12$ & $111 \pm 13$ & $115 \pm 17$ & $112 \pm 11$ & $121 \pm 19$ & $112 \pm 13$ & $124 \pm 14$ \\
\hline Jeûne j 2 & $112 \pm 11^{\mathrm{a}}$ & $118 \pm 14^{\mathrm{ab}}$ & $107 \pm 17^{\mathrm{a}}$ & $118 \pm 13^{\mathrm{ab}}$ & $106 \pm 9^{\mathrm{a}}$ & $127 \pm 15^{b}$ & $114 \pm 12^{a b}$ \\
\hline \multicolumn{8}{|l|}{ Méthionine } \\
\hline Nourries & $18 \pm 2^{\mathrm{a}}$ & $18 \pm 3^{\mathrm{a}}$ & $21 \pm 4^{a c}$ & $27 \pm 3^{b}$ & $25 \pm 3^{b c}$ & $21 \pm 3^{a c}$ & $26 \pm 2^{b}$ \\
\hline Jeûne j 1 & $17 \pm 3^{\mathrm{ab}}$ & $2^{\mathrm{ac}}$ & 16 & $18 \pm 3^{\mathrm{ab}}$ & $19 \pm 3^{a b}$ & $23 \pm 5^{\mathrm{c}}$ & $19 \pm 3^{a b}$ \\
\hline Jeûne j 2 & 15 & 1 & $11 \pm 2^{b}$ & $16 \pm 3^{\mathrm{ac}}$ & $19 \pm 2^{c}$ & $17 \pm 2^{\mathrm{cd}}$ & $14 \pm 3^{\mathrm{a}}$ \\
\hline \multicolumn{8}{|c|}{ Phénylalanine } \\
\hline Nourries & $48 \pm 4^{a b}$ & $43 \pm 7^{\mathrm{a}}$ & $51 \pm 8^{\mathrm{bc}}$ & $56 \pm 8^{c}$ & $58 \pm 9^{c e}$ & $63 \pm 8^{\mathrm{de}}$ & $65 \pm 6^{\mathrm{d}}$ \\
\hline Jeûne j & 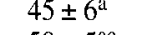 & & $55 \pm 6^{\mathrm{b}}$ & $49 \pm$ & $38 \pm 6^{c}$ & 38 & $45 \pm 6^{\mathrm{a}}$ \\
\hline Jeûne $\mathrm{j} 2$ & $50 \pm 5^{a c}$ & $38 \pm 4^{b}$ & $50 \pm 6^{a c}$ & $51 \pm 7^{\mathrm{ac}}$ & $45 \pm 7^{a b}$ & $54 \pm 7^{c}$ & $49 \pm 7^{\mathrm{ac}}$ \\
\hline \multicolumn{8}{|l|}{ Thréonine } \\
\hline Nourries & $73 \pm 6^{a}$ & $86 \pm 9^{\mathrm{ab}}$ & $87 \pm 9^{\mathrm{ab}}$ & $92 \pm 7^{b}$ & $80 \pm$ & $70 \pm$ & $57 \pm 4^{\mathfrak{c}}$ \\
\hline Jeû & $+6^{a}$ & $=11^{\text {bd }}$ & 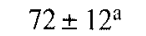 & $84 \pm 9^{\text {ad }}$ & $103 \pm 17^{b}$ & & $91 \pm 9^{b d}$ \\
\hline Jeûne j 2 & $70 \pm 5^{\mathrm{ab}}$ & $76 \pm 11^{b c}$ & $77 \pm 10^{b}$ & $64 \pm 8^{a}$ & $76 \pm 6^{b c}$ & $83 \pm 5^{c}$ & $67 \pm 6^{a}$ \\
\hline \multicolumn{8}{|l|}{ Tyrosinel } \\
\hline Nourries & $42 \pm 4^{\mathrm{a}}$ & $55 \pm 9^{b}$ & $41 \pm 7^{\mathrm{ad}}$ & $50 \pm 6^{a}$ & $33 \pm 5^{\text {cd }}$ & $34 \pm 4^{\text {cd }}$ & $29 \pm 1^{c}$ \\
\hline Jeûne j 1 & $32 \pm 6^{a c}$ & $38 \pm 3^{\mathrm{ab}}$ & $36 \pm 3^{\mathrm{ab}}$ & $41 \pm 7^{b}$ & $36 \pm 4^{\mathrm{ab}}$ & $29 \pm 4^{\mathrm{c}}$ & $31 \pm 5^{\mathrm{ac}}$ \\
\hline Jeûne j 2 & $29 \pm 3^{\text {ab }}$ & $35 \pm 7^{\mathrm{a}}$ & $31 \pm 7^{a c}$ & $30 \pm 3^{a b}$ & $23 \pm 4^{b c}$ & $23 \pm 4^{b c}$ & $22 \pm 4^{b}$ \\
\hline \multicolumn{8}{|l|}{ Valine } \\
\hline Nourries & $176 \pm 20^{\mathrm{a}}$ & $208 \pm 26^{b c}$ & $198 \pm 26^{a b}$ & $232 \pm 22^{c d}$ & $228 \pm 24^{\mathrm{cd}}$ & $225 \pm 14^{\mathrm{cd}}$ & $245 \pm 24^{\mathrm{d}}$ \\
\hline Jeûne j 1 & $193 \pm 20^{\mathrm{a}}$ & $212 \pm 25^{a b}$ & $230 \pm 30^{\mathrm{bc}}$ & $254 \pm 25^{c}$ & $229 \pm 20^{b c}$ & $245 \pm 33^{c}$ & $228 \pm 20^{b c}$ \\
\hline Jeûne j 2 & $249 \pm 23$ & $244 \pm 18$ & $236 \pm 25$ & $251 \pm 19$ & $260 \pm 24$ & $256 \pm 24$ & $241 \pm 31$ \\
\hline
\end{tabular}

Les repas ont été distribués à $6 \mathrm{~h} 15$ et à $15 \mathrm{~h} 30$. ' Acide aminé habituellement désigné sous le nom d'acide aminé semi-essentiel. ${ }^{a, b, c, d, e}$ Les moyennes dans une même ligne sans lettre commune sont différentes $(p<0,05)$. 
sation des réserves corporelles (lipides, protéines).

Mais, contrairement aux observations faites chez la vache tarie (Ndibualonji et al, 1992), les $2 \mathrm{j}$ de jeûne n'ont pas significativement augmenté les teneurs plasmatiques en Ala et Gln, ce qui est en accord avec les observations de Lomax et Baird (1983). Il est raisonnable de penser que ce maintien des teneurs plasmatiques en Ala et Gln vient du fait que l'Ala et la Gln, qui sont les principaux AA glucoformateurs chez les ruminants, sont libérés du muscle pendant le jeûne en plus grande quantité que celle prélevée par le foie et le rein pour la synthèse du glucose (Wolff et al, 1972 ; Lomax et Baird, 1983 ; Ndibualonji et Godeau, 1993). En effet, la plupart des AA libérés par le muscle ne sont pas directement transportés au foie, mais sont en premier lieu convertis dans le muscle même en Ala et Gln, ces deux AA étant les principaux transporteurs des chaînes carbonées et de l'azote provenant des autres $\mathrm{AA}$ du muscle vers le foie où les chaînes carbonées sont converties en glucose et l'azote en urée (Wolff et al, 1972 ; Heitmann et Bergman, 1980 ; Teleni, 1993). Le maintien des teneurs plasmatiques en Ala et Gln pourrait également provenir du fait que ces deux AA ne sont pas prélevés du sang par la glande mammaire de la vache en grande quantité (Wohlt et al, 1977 ; Peeters et al, 1979). En revanche, la Gly et la Ser qui sont également prélevées en petite quantité par la glande mammaire (Wohlt et al, 1977) ont augmenté dans le plasma au cours du jeûne. Ces deux AA qui jouent également le rôle de transporteur (Wolff et al, 1972) seraient moins glucogéniques que l'Ala et la Gln chez la vache (Ndibualonji et Godeau, 1993).

Le maintien des teneurs en Ile et en Val après $2 \mathrm{j}$ de jeûne est également en contradiction avec les observations faites chez le mouton (Bergman et Pell, 1983 ; Bergen et al, 1988) et chez la vache tarie (Ndibualonji et $\mathrm{al}, 1992$ ) où ces AA augmentent dans le plasma. Il est possible que cela découle du métabolisme de la glande mammaire. En effet, l'Ile et la Val sont prélevés du sang de la vache en quantité plus importante que celle sécrétée dans les protéines du lait (Wohlt et al, 1977). Il se peut aussi que la néoglucogenèse à partir de l'Ile et de la Val ait augmenté au cours du jeûne car ils sont également glucogéniques chez la vache (Ndibualonji et Godeau, 1993). En revanche, l'Arg et la Leu qui sont également prélevées en grande quantité par la glande mammaire (Wohlt et al, 1977 ; Peeters et al, 1979) ont augmenté dans le sang au $2^{\mathrm{e}}$ jour de jeûne, ce qui peut être dû à la diminution significative $(P<0,05)$ de la production laitière, et donc de la synthèse des protéines du lait, observée au cours du $2^{\mathrm{e}}$ jour de jeûne. En outre, la Leu n'intervient pas dans la néoglucogenèse hépatique car elle est cétogénique à $100 \%$ (Ndibualonji et Godeau, 1993 ; Ndibualonji, 1995) et les vitesses de décarboxylation, de désamination et de transamination de cet AA diminuent pendant le jeûne (Wijayasinghe et al, 1983 ; Bergen et al, 1988). Comme pour la Leu, l'augmentation de la concentration plasmatique en Lys au cours du jeûne peut être due au fait que cet AA n'est pas glucogénique chez les ruminants (Ndibualonji et Godeau, 1993).

La mobilisation protéique et la néoglucogenèse AA-dépendante apparemment plus intenses lors du $2^{\mathrm{e}}$ que du $1^{\mathrm{er}}$ jour de jeûne n'ont pas empêché les valeurs de la glycémie de chuter significativement. Cela rejoint les observations similaires faites chez la vache haute productrice soumise au jeûne (Baird et al, 1972 ; Fröhli et Blum, 1988) et peut s'expliquer par le fait que l'oxydation et le transfert de chaînes carbonées du glucose dans le lactose du lait, qui représentent environ $80 \%$ de l'utilisation totale du glucose chez la vache (Bickerstaffe et al, 1974), étaient restés importants tandis que la néoglucogenèse à partir des substrats endogènes (AA, glycérol, lactate) était devenue insuffisante pour assurer l'homéostasie gluci- 
dique. En outre, le glucose est requis par la glande mammaire pour la synthèse de la matière grasse du lait, notamment en intervenant dans la synthèse du glycérol avec lequel les acides gras sont estérifiés et en fournissant les équivalents réducteurs sous la forme de NADPH pour la lipogenèse mammaire (Bines et Hart, 1982). Cette chute de la glycémie est probablement responsable de la diminution significative de la production laitière au $2^{\mathrm{e}}$ jour de jeûne. En effet, la chute de la glycémie entraîne une chute du lactose, élément osmorégulateur qui règle le volume cellulaire du lait (Kronfeld, 1982 ; Sutton, 1989).

\section{Évolutions journalières}

En accord avec les résultats rapportés chez la vache en lactation (Kunz et al, 1985) et chez la vache tarie (Ndibualonji et al, 1995) sur les profils journaliers de l'aminoacidémie libre, nous avons observé la concentration la plus basse pour l'Ala, l'Arg, la Gly, l'His, l'Ile, la Leu, la Met, la Pro et la Val 1 heure environ après la fin du repas du matin. Cela peut s'expliquer par une réduction de la dégradation protéique dans les muscles, comme l'indiquent le bas niveau de la teneur plasmatique en MeHis enregistré à $9 \mathrm{~h}$ dans la présente étude chez la vache en lactation ainsi que la moindre quantité de $\mathrm{MeHis}$ excrétée dans les urines et le niveau élevé de l'insulinémie déterminés à ce moment de la journée chez la vache tarie alimentée aux mêmes heures (Ndibualonji et al, 1995). En revanche, pour certains AA (Asp, Asn, Gln, Glu, Thr et Tyr), la concentration la plus basse a été observée aux heures les plus éloignées de la fin du repas du matin. Cette chute s'expliquerait par une utilisation accrue de ces AA à des fins énergétiques. En effet, un déficit énergétique, apprécié par la diminution des teneurs ruminales en acides gras volatils, a été rapporté entre les repas chez la vache tarie nourrie deux fois par jour (Ndibualonji, 1995 ; Ndibualonji et al, 1995). La mobilisation des protéines musculaires entre les repas est mise en évidence par l'augmentation progressive de la MeHis du plasma de $12 \mathrm{~h}$ à $15 \mathrm{~h}$ et par le fait que la concentration la plus élevée pour l'Ala, l'Arg, la Gly, l'His, l'Ile, la Phe et la Val a été observée à $15 \mathrm{~h}$ (tableaux IV, V). Le fait que l'Ala, qui est le premier AA glucoformateur chez la vache (Ndibualonji et Godeau, 1993), ait progressivement augmenté entre les repas plutôt que de diminuer corrobore la non-variation des niveaux moyens de cet AA pendant le jeûne (tableau II) et confirme que l'Ala est libéré par le muscle en plus grande quantité que celle prélevée par le foie pour la synthèse du glucose pendant les périodes de déficit énergétique (Heitmann et Bergman, 1980). De même, le fait que les concentrations plasmatiques en Thr et Tyr aient diminué au fur et à mesure qu' on s'éloigne du dernier repas est en accord avec la diminution significative des niveaux moyens de ces deux AA observée le $2^{\mathrm{e}}$ jour de jeûne (tableau II). Par ailleurs, contrairement aux informations obtenues chez la vache tarie (Ndibualonji et al, 1995), très peu de variations journalières de la glycémie ont été observées dans le présent essai sur la vache en lactation. Cela rejoint les observations faites par Manston et al (1981) chez la vache en lactation nourrie et traite deux fois par jour $(7 \mathrm{~h} 15$ et $14 \mathrm{~h} 15$ ) et produisant $14 \mathrm{~L}$ de lait par jour où $2 \%$ seulement des variations journalières de la glycémie ont été observés.

Enfin, la concentration la plus élevée en urée plasmatique a été observée à $9 \mathrm{~h}$ du matin, rejoignant en cela nos observations antérieures chez la vache tarie (Ndibualonji et al, 1995 ; Dehareng et al, 1996) où la concentration la plus élevée du nycthémère était enregistrée aux environs de $8 \mathrm{~h}$ et $9 \mathrm{~h}$.

En situation de jeûne, des modifications intrajournalières dans les concentrations plasmatiques des paramètres métaboliques étudiés ont également été observées, mais celles-ci ont été dans l'ensemble plus faibles 
que celles observées en situation nourrie, et en particulier pour la glycémie.

\section{CONCLUSION}

Nos résultats montrent que, comme chez la vache tarie, la vache en milieu de lactation mobilise rapidement ses protéines musculaires au cours du jeûne. Cependant, contrairement à la vache tarie, le mécanisme de la néoglucogenèse ne permet d'assurer l'homéostasie glucidique qu'au cours des 24 premières heures de jeûne. La chute de la glycémie à partir du $2^{\mathrm{e}}$ jour de jeûne s'accompagne d'une chute de la production laitière, démontrant que la mobilisation des réserves corporelles pendant le jeûne ne permet pas de maintenir la production laitière, même chez une vache en milieu de lactation. Si les variations journalières de l'aminoacidémie libre, de l'urémie et de la glycémie sont plus importantes en situation nourrie qu'en situation de jeûne, la glycémie varie finalement très peu au cours de la journée chez la vache en lactation.

\section{REMERCIEMENTS}

Les auteurs tiennent à remercier $\mathrm{L}$ Trzpiot pour l'assistance technique. Ce travail a été supporté en partie par la fondation A Seghers (place du 20-Août, 7, bât A1, B-4000, Liège).

\section{RÉFÉRENCES}

Andrieu J, Demarquilly C, Sauvant D (1988) Tables de la valeur nutritive des aliments. In : Alimentation des Bovins, Ovins et Caprins (R Jarrige, éd). Inra Publ, Paris, 351-443

Baird GD, Heitzman RJ, Hibbit KG (1972) Effects of starvation on intermediary metabolism in the lactating cow. Biochem $J$ 128, 1311-1318

Baldwin RL, Kim WY (1993) Lactation. In : Quantitative aspects of ruminant digestion and metabolism (JM Forbes, J France, eds). CAB International, Wallingford, Royaume-Uni, 433-451

Bergen WG, Busboom JR, Merkel RA (1988) Leucine degradation in sheep. BrJ Nutr 59, 323-333
Bergman EN, Pell JM (1983) Leucine metabolism in tissues of fed and starved sheep. Fed Proc 41 (Suppl), 343

Bickerstaffe R, Annison EF, Linzell JL (1974) The metabolism of glucose, acetate, lipids and amino acids in lactating dairy cows. J Agric Sci Camb 82, $71-85$

Bines JA, Hart IC (1982) Metabolic limits to milk production, especially roles of growth hormone and insulin. J Dairy Sci 65, 1375-1389

Bouchat JC, Doizé F, Paquay R (1981) Influence of diet and prolonged fasting on blood lipids, ketone bodies, glucose and insulin in adult sheep. Reprod Nutr Dev 21, 69-81

Dehareng D, Godeau JM (1991) The duration of masticating activities and the feed energetic utilization of Friesian lactating cows on maize silage-based rations. J Anim Physiol Anim Nutr 65, 194-205

Dehareng D, Ndibualonji BB, Godeau JM (1996) Continuous profiles of ruminal ammonia and plasma urea in dry Friesian cows on hay-based rations. J Anim Physiol Anim Nutr 75, 57-72

Fröhli DM, Blum JW (1988) Nonesterified fatty acids and glucose in lactating dairy cows: diurnal variations and changes in responsiveness during fasting to epinephrine and effects of beta-adrenergic blockade. $J$ Dairy $S c i 71,1170-1177$

Hawkins RA, Williamson DH, Krebs HA (1971) Ketone-body utilization by adult and suckling rat brain in vivo. Biochem $J$ 122, I3-18

Heitmann RN, Bergman EN (1980) Integration of amino acid metabolism in sheep: effect of fasting and acidosis. Am J Physiol 239, E248-E254

Koenig JM, Boling JA (1980) Plasma amino acid profiles, urea and ammonia concentrations in fasted ewe, as influenced by age. Nutr Rep Int 22, 101 108

Kronfeld DS (1982) Major metabolic determinants of milk volume, mammary efficiency, and spontaneous ketosis in dairy cows. J Dairy Sci $65,2204-$ 2212

Kunz PL, Blum JW, Hart IC, Bickel H, Landis J (1985) Effects of different energy intakes before and after calving on food intake, performance and blood hormones and metabolites in dairy cows. Anim Prod 40, 219-231

Leibholz J (1970) The effect of starvation and low nitrogen intakes on the concentration of free amino acids in the blood plasma and on the nitrogen metabolism in sheep. Aust J Agric Res 21, 723-734

Lindsay DB, Setchell BP (1976) The oxydation of glucose, ketone bodies and acetate by the brain of normal and ketonaemic sheep. J Physiol 259, 801-823

Lomax MA, Baird GD (1983) Blood flow and nutrient exchange accoss the liver and gut of the dairy cow. Br J Nutr 49, 481-496

Manston R, Rowlands GJ, Little W, Collis KA (1981) Variability of time blood composition of dairy cows 
in relation to time of day. J Agric Sci Camb 96 , 593-598

Nagasawa T, Hashiguchi N, Onodera R (1993) Effect of starvation on the plasma $\mathrm{N}^{\mathrm{r}}$-methylhistidine in goats. Biosci Biotech Biochem 57 (Suppl), 517

Ndibualonji BB (1995) Implications des protéines de réserve dans les profils continus de l'aminoacidémie libre, de l'urémie et de la glycémie chez la vache en tarissement et en lactation. Thèse de doctorat en sciences vétérinaires, université de Liège, Belgique

Ndibualonji BB, Godeau JM (1993) La néogłıcogenèse et les acides aminés chez les ruminants : revue. Ann Méd Vét 137, 537-554

Ndibualonji BB, Dehareng D, Godeau JM (1995) La sous-alimentation énergétique et/ou azotée chez la vache tarie. Effets sur les acides gras volatils du rumen, quelques métabolites et hormones du plasma et l'excrétion urinaire de la 3-méthylhistidine. Reprod Nutr Dév 35, 137-154

Ndibualonji BB, Dehareng D, Debue P, Godeau JM (1992) Détermination des acides aminés libres du plasma par HPLC et étude des effets du jeûne sur l'aminoacidémie libre chez la vache Frisonne tarie. Ann Méd Vét 136, 413-422

Ndibualonji BB, Debue P, Dehareng D, Godeau JM (1993) Effect of short-term fasting on plasma concentrations of amino acids, glucose and insulin in non-pregnant and non-lactating Friesian cows. Ann Zootech 42 (Suppl), 212

Peeters G, Houvenaghel A, Roets E, Massart-Leën AM, Verbeke R, Dhondt G, Verschooten F (1979) Electromagnetic blood flow recording and balance of nutrients in the udder of lactating cows. $J$ Anim Sci $48,1143-1153$

Pell JM, Bergman EN (1983) Cerebral metabolism of amino acids and glucose in fed and fasted sheep. Am J Physiol 224, E282-E289

SAS (1989) SAS User's guide: Statistics. Version 6, 4th ed. SAS Inst Inc, Cary, NC

Schmidt FH (1961) Die enzymatische Bestimmung von Glucose und Fructose nebeneinander. Klin Wsch 39, 1244-1247

Sutton JD (1989) Altering milk composition by feeding. J Dairy Sci 72, 2801-2814

Teleni E (1993) Catabolism and synthesis of amino acids in skeletal muscle : their significance in monogastric Mammal and Ruminants. Aust J Agric Res 44, 443-461

Weiner RH, Hirsch HJ, Spitzer JJ (1971) Cerebral extraction of ketones and their penetration into CSF in the dog. Am J Physiol 220, 1542-1546

Wijayasinghe MS, Milligan LP, Thompson JP (1983) In vitro degradation of leucine in muscle, adipose tissue, liver, and kidney of fed and starved sheep. Biosci Rep 3, 1133-1140

Wohlt JE, Clark JH, Derrig RG, Davis CL (1977) Valine, leucine, and isoleucine metabolism by lactating bovine mammary tissue. J Dairy Sci 60, 1875-1882

Wolff JE, Bergman EN, Williams HH (1972) Net metabolism of plasma amino acid by liver and portaldrained viscera of fed sheep. Am J Physiol 223, 438-446 